\title{
Temporal Pattern of Cocaine Intake Determines Tolerance vs Sensitization of Cocaine Effects at the Dopamine Transporter
}

\author{
Erin S Calipari', Mark J Ferris', Benjamin A Zimmer', David CS Roberts' and Sara R Jones*,' \\ 'Department of Physiology and Pharmacology, Wake Forest School of Medicine, Winston-Salem, NC, USA
}

\begin{abstract}
The dopamine transporter (DAT) is responsible for terminating dopamine (DA) signaling and is the primary site of cocaine's reinforcing actions. Cocaine self-administration has been shown previously to result in changes in cocaine potency at the DAT. To determine whether the DAT changes associated with self-administration are due to differences in intake levels or temporal patterns of cocaineinduced DAT inhibition, we manipulated cocaine access to produce either continuous or intermittent elevations in cocaine brain levels. Long-access (LgA, 6h) and short-access (ShA, $2 \mathrm{~h}$ ) continuous self-administration produced similar temporal profiles of cocaine intake that were sustained throughout the session; however, LgA had greater intake. ShA and intermittent-access (IntA, $6 \mathrm{~h}$ ) produced the same intake, but different temporal profiles, with 'spiking' brain levels in IntA compared with constant levels in ShA. IntA consisted of 5-min access periods alternating with 25 -min timeouts, which resulted in bursts of high responding followed by periods of no responding. DA release and uptake, as well as the potency of cocaine for DAT inhibition, were assessed by voltammetry in the nucleus accumbens slices following control, IntA, ShA, and LgA self-administration. Continuous-access protocols (LgA and ShA) did not change DA parameters, but the 'spiking' protocol (IntA) increased both release and uptake of DA. In addition, high continuous intake ( $L g A)$ produced tolerance to cocaine, while 'spiking' (IntA) produced sensitization, relative to ShA and naive controls. Thus, intake and pattern can both influence cocaine potency, and tolerance seems to be produced by high intake, while sensitization is produced by intermittent temporal patterns of intake. Neuropsychopharmacology (2013) 38, 2385-2392; doi:I0.1038/npp.20 I3.136; published online 19 June 2013
\end{abstract}

Keywords: self-administration; cocaine; voltammetry; dopamine; dopamine transporter; nucleus accumbens

\section{INTRODUCTION}

A substantial literature has documented the effects of cocaine on the dopamine (DA) system and its involvement in the addiction process (Roberts et al, 1977; Izenwasser, 2004; Stuber et al, 2011; Blum et al, 2012). Cocaine exerts its reinforcing and neurochemical effects by inhibiting the dopamine transporter (DAT), which leads to augmented DA signaling (Ritz et al, 1987; Kristensen et al, 2011). DA elevations in the nucleus accumbens (NAc) core region are critically involved in self-administration behaviors, reinstatement, cue-reward association, assignment of motivational value, and goal-oriented behaviors (for a review, see Wheeler and Carelli, 2009; Kalivas and McFarland, 2003; Aragona et al, 2009; Willuhn et al, 2010).

Both tolerance and sensitization can develop to the neurochemical effects of cocaine; however, the nature of these changes appears to depend on the dosing parameters used. An extensive literature, primarily using daily experimenteradministered intraperitoneal injections has documented

*Correspondence: Dr SR Jones, Department of Physiology and Pharmacology, Wake Forest School of Medicine, Medical Center Boulevard, Winston-Salem, NC 27I57, USA,

Tel: + 336 716 8533, Fax: + I 336 716 850।,

E-mail: srjones@wakehealth.edu

Received 27 March 20 I3; revised IO May 20 I3; accepted II May 20 I3; accepted article preview online 30 May 2013 neurochemical sensitization associated with the mesolimbic DA system (Kalivas and Duffy, 1990, 1993; Parsons and Justice, 1993; Addy et al, 2010). Sensitization of cocaineinduced increases in extracellular DA has been linked not only to alterations in the regulation of DA release and reuptake (Jones et al, 1996; Addy et al, 2010), but also to influences such as glutamate (for a review, see Vanderschuren and Kalivas, 2000), GABA (for a review, see Steketee, 2005; Filip et al, 2006), and serotonin (Neumaier et al, 2002; Filip et al, 2010). By contrast, studies using self-administration procedures have generally shown tolerance to cocaine's DA-elevating and DAT-inhibiting effects. Tolerance to the DA-elevating effects of cocaine, as measured by microdialysis (Hurd et al, 1989; Mateo et al, 2005; Lack et al, 2008; Ferris et al, 2011). It is possible that cocaine tolerance is due to a reduced ability of cocaine to inhibit the DAT, which has been reported in a number of paradigms where cocaine levels are high and sustained over daily extended-access self-administration sessions (Mateo et al, 2005; Ferris et al, 2011, 2012; Calipari et al, 2012).

Therefore, experimenter-delivered and self-administration protocols produce distinctly different effects on the DA system. There are many differences between these protocols including contingency, temporal pattern of intake, total consumption, and route of administration, which may influence the divergent neurochemical consequences. For example, experimenter-administered injections result 
in an intermittent pattern of drug administration, whereas intravenous self-administration procedures result in high rates of responding and high levels of administration for many hours (ie, for the duration of the self-administration session).

In this study, we investigated the contribution of (1) pattern of consumption and (2) total intake of cocaine on rapid DA signals in the NAc and the ability of self-administered cocaine to either sensitize or induce tolerance to the pharmacological effects of cocaine on the DAT. Self-administration paradigms were used to control for the contribution of contingency and route of administration on the effects of the DA system. A long-access $(\operatorname{LgA})$ procedure was used in which cocaine levels were sustained for $6 \mathrm{~h}$ each day (Ahmed et al, 2003; Martin et al, 2011; Peraile et al, 2010). This was compared with an intermittent-access (IntA) self-administration procedure in which cocaine was available during a 6-h session but only during 5 -min trials, which were separated by 25 -min timeouts. This protocol allows animals to 'load up' during each trial, but ensures that cocaine levels cannot be maintained. The IntA procedure, which results in a 'spiking' pattern of intake, has recently been shown to produce a greater augmentation of the reinforcing effects of cocaine compared with the long-access ( LgA) procedure (Zimmer et al, 2012). We also included a short-access (ShA) group in which cocaine levels were matched to the IntA group but were maintained during a 2 -h session similar to LgA. Here we demonstrate that intake and pattern can both influence cocaine potency at the DAT; while tolerance seems to be dictated by total intake, sensitization appears to be determined by pattern.

\section{MATERIALS AND METHODS}

\section{Animals \\ Male Sprague-Dawley rats (375-400 g; Harlan Laboratories, Frederick, MD) were maintained on a $12: 12 \mathrm{~h}$ reverse light/ dark cycle (0300 hours lights off; 1500 hours lights on) with food and water ad libitum. All animals were maintained according to the National Institutes of Health guidelines in Association for Assessment and Accreditation of Laboratory Animal Care accredited facilities. The experimental protocol was approved by the Institutional Animal Care and Use Committee at Wake Forest School of Medicine.}

\section{Self-Administration}

Rats were anesthetized and implanted with chronic indwelling jugular catheters as described previously (Liu et al, 2007). Animals were singly housed, and all sessions took place in the home cage during the active/dark cycle (0900-1500 hours). After a 2-day recovery period, animals underwent a training paradigm within which animals were given access on a fixed ratio one (FR1) schedule to a cocaine-paired lever, whereby a single lever press initiated an intravenous injection of cocaine $(0.75 \mathrm{mg} / \mathrm{kg}$, infused over $4 \mathrm{~s}$ ). After each response/infusion, the lever was retracted and a stimulus light was illuminated for a 20 -s timeout period. Training sessions were terminated after a maximum of 20 infusions or $6 \mathrm{~h}$, whichever occurred first. Acquisition occurred when an animal responded to 20 injections for two consecutive days and a stable pattern of infusion intervals was present. Following training, animals were assigned to either ShA, IntA, or LgA groups. All self-administration was 14 consecutive sessions, after which animals were killed and brains were prepared for voltametric recordings.

\section{Control}

Controls were naive rats housed in the same conditions as animals undergoing self-administration.

\section{LgA Group}

Subjects completed daily 6-h sessions during which they had unlimited access to cocaine $(0.75 \mathrm{mg} / \mathrm{kg}$; infused over $4 \mathrm{~s}$ ) on an FR1 schedule for 14 consecutive days. Upon each infusion, the lever was retracted, and a stimulus light signaled a 20 -s timeout period.

\section{ShA Group}

Subjects were given access to cocaine $(0.75 \mathrm{mg} / \mathrm{kg}$; infused over $4 \mathrm{~s}$ ) on an FR1 schedule during 2-h daily sessions for 14 consecutive days. At the start of each infusion, a stimulus light signaled a 20 -s timeout period during which the lever was retracted.

\section{IntA Group}

Subjects were given access to cocaine on an intermittent schedule of administration described previously (Zimmer et al, 2012). Briefly here, during each 6-h session animals had access to cocaine for 12 five-minute trails separated by 25-min timeout periods. Within each 5-min session, there were no timeouts other than during each infusion, and the animal could press the lever on an FR1 schedule to receive a 1 -s infusion of cocaine $(0.375 \mathrm{mg} / \mathrm{kg}$ per infusion).

\section{In Vitro Voltammetry}

Fast scan cyclic voltammetry (FSCV) was used to characterize presynaptic DA system kinetics and the ability of psychostimulants to inhibit DA uptake in the NAc. Voltammetry experiments were conducted during the dark phase of the light/dark cycle, $18 \mathrm{~h}$ after commencement of the final self-administration session. A vibrating tissue slicer was used to prepare $400-\mu \mathrm{m}$-thick coronal brain sections containing the NAc. The tissue was immersed in oxygenated artificial cerebrospinal fluid (aCSF) containing (in $\mathrm{mM}$ ): $\mathrm{NaCl}$ (126), $\mathrm{KCl}(2.5), \mathrm{NaH}_{2} \mathrm{PO}_{4}$ (1.2), $\mathrm{CaCl}_{2}$ (2.4), $\mathrm{MgCl}_{2}$ (1.2), $\mathrm{NaHCO}_{3}$ (25), glucose (11), L-ascorbic acid (0.4) and $\mathrm{pH}$ was adjusted to 7.4. Once sliced, the tissue was transferred to the testing chambers containing bath aCSF $\left(32{ }^{\circ} \mathrm{C}\right)$, which flowed at $1 \mathrm{ml} / \mathrm{min}$. A carbon fiber microelectrode $(100-200 \mu \mathrm{M}$ length, $7 \mu \mathrm{M}$ radius) and bipolar stimulating electrode were placed into the core of the NAc, which was selected because of its role in the reinforcing and rewarding actions of cocaine. DA release was evoked by a single electrical pulse ( $300 \mu \mathrm{A}, 4 \mathrm{~ms}$, monophasic) applied to the tissue every $5 \mathrm{~min}$. Extracellular DA was recorded by 
applying a triangular waveform $(-0.4$ to +1.2 to $-0.4 \mathrm{~V}$ vs $\mathrm{Ag} / \mathrm{AgCl}, 400 \mathrm{~V} / \mathrm{s})$. Once the extracellular DA response was stable, cocaine $(0.3-30 \mu \mathrm{mol} / \mathrm{l})$ was applied cumulatively to the brain slice.

\section{Data Analysis}

Demon Voltammetry and Analysis Software was used for all analyses of FSCV data (Yorgason et al, 2011). To evaluate DA kinetics and drug potency, evoked levels of DA were modeled using Michaelis-Menten kinetics. Apparent $K_{\mathrm{m}}$ (app. $K_{\mathrm{m}}$ ) was used as a measure of the ability of cocaine to inhibit DA clearance, and to evaluate changes in cocaine potency. As app. $K_{\mathrm{m}}$ increases, the affinity of DA for the DAT decreases. Increasing concentrations of cocaine linearly decrease the affinity of DA for the DAT, such that shifts in app. $K_{\mathrm{m}}$ across treatment groups indicate shifts in the ability of cocaine to inhibit DA uptake. Recording electrodes were calibrated by recording responses (in electrical current; $\mathrm{nA})$ to a known concentration of DA $(3 \mu \mathrm{M})$ using a flow-injection system.

\section{Calculating $K_{\mathbf{i}}$ Values}

Inhibition constants $\left(K_{\mathrm{i}}\right)$ were determined by plotting the linear concentration-effect profiles and determining the slope of the linear regression. The $K_{\mathrm{i}}$ was calculated by the equation $K_{\mathrm{m}}$ /slope. $K_{\mathrm{i}}$ values are reported in $\mu \mathrm{M}$ and are a measure of the drug concentration that is necessary to produce $50 \%$ uptake inhibition. $K_{\mathrm{i}}$ is a measure of the drug affinity for the DAT, reported in concentration of drug, while app. $K_{\mathrm{m}}$ is a DA-related parameter that estimates DA uptake inhibition.

\section{Statistics}

Graph Pad Prism (version 5; Graph Pad Software, La Jolla, CA) was used to statistically analyze data and create graphs. Baseline voltammetry data and $K_{\mathrm{m}}$ values were compared using a one-way analysis of variance (ANOVA). When main effects were obtained $(p<0.05)$, differences between groups were tested using Tukey's post hoc tests. Release data and data obtained after perfusion of cocaine were subjected to a two-way ANOVA, with the experimental group and concentration of drug as the factors. Differences between groups were tested using a Bonferroni post hoc test. Given the lack of a Gaussian distribution for $K_{\mathrm{i}}$ values, non-parametric tests (Kruskal-Wallis and Neuman-Keuls) were used to compare inhibition constants.

\section{RESULTS}

\section{ShA and LgA Result in Similar Pattern of Self-Administration that is Different from IntA}

Figure 1a contains representative behavioral plots demonstrating the different patterns of self-administration behavior between ShA, LgA, and IntA. We demonstrated that ShA results in high and consistent rates of responding for cocaine over a 2 -h self-administration session (Figure 1a, top panel). IntA results in an intermittent pattern of responding for cocaine, characterized by high rates
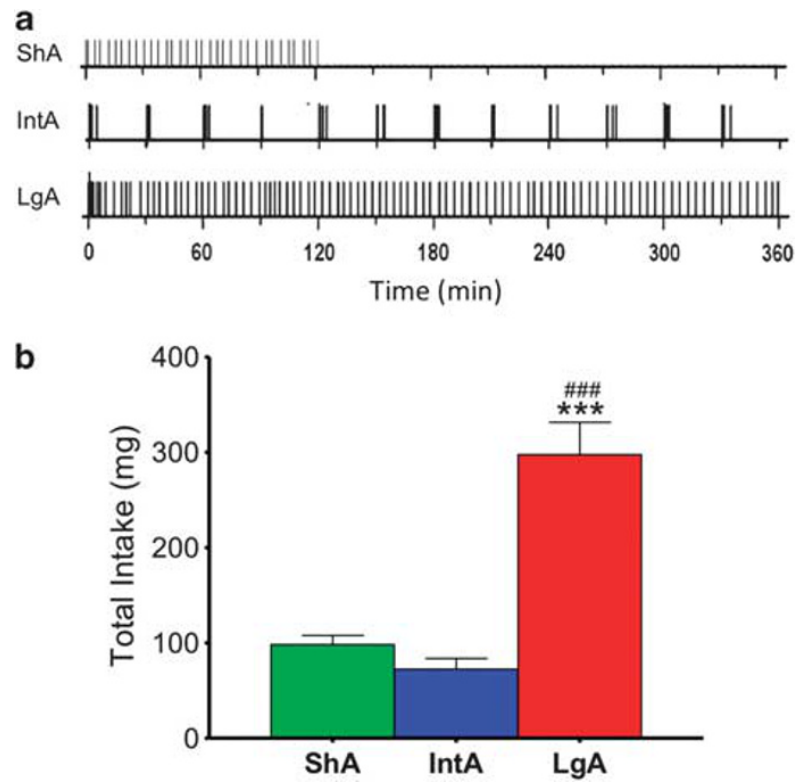

Figure I Short-access (ShA), Intermittent-access (IntA), and long-access ( $\operatorname{gA} A)$ cocaine self-administration result in different patterns of selfadministration. (a) Plots demonstrating representative self-administration behavior throughout each session for individual rats self-administering cocaine. Tick marks represent infusions/responses on the lever earned on a fixed ratio one schedule of reinforcement. (a, top) ShA results in high consistent rates of responding over the $2-h$ session. (a, middle) IntA is achieved by giving 5 -min access followed by 25 -min forced timeouts. This results in bursts of high responding followed by no responding. (a, bottom) LgA results in high consistent rates of responding over the 6-h session. (b) Group data plotting total intake over the 14 days of self-administration for each paradigm. ${ }^{*} * * P<0.00$ I vs ShA; ${ }^{\# \# \#} p<0.00$ I vs IntA.

of responding followed by timeout periods within which no responding occurs (Figure 1a, middle panel). LgA results in high and consistent rates of responding, similar to responding for ShA, for cocaine over a 6-h self-administration session (Figure 1a, bottom panel).

\section{LgA Results in Greater Cocaine Intake as Compared With ShA and IntA}

One-way ANOVA revealed a main effect of intake between groups $\left(\mathrm{F}_{2,11}=39.28, p<0.0001\right.$; Figure $\left.1 \mathrm{~b}\right)$. Tukey's post hoc analysis indicated that there was a significant increase in intake for the LgA group $v s$ both the ShA group $(p<0.0001)$ and the IntA group $(p<0.0001)$. Further, although the pattern of self-administration differed, the total cocaine intake between the ShA and IntA groups did not differ significantly. Because ShA results in similar intake to the IntA group and similar pattern of estimated cocaine brain levels to the LgA group, it was used as a comparator group to determine if effects were due to total intake or temporal pattern of cocaine self-administration.

\section{IntA, but not LgA or ShA, Increases Stimulated DA Release and $V_{\max }$}

FSCV was used to assess presynaptic baseline DA system kinetics. ANOVA indicated a main effect of paradigm on stimulated DA release $\left(F_{3,63}=4.020, p<0.05\right.$; Figures $2 \mathrm{a}$ 


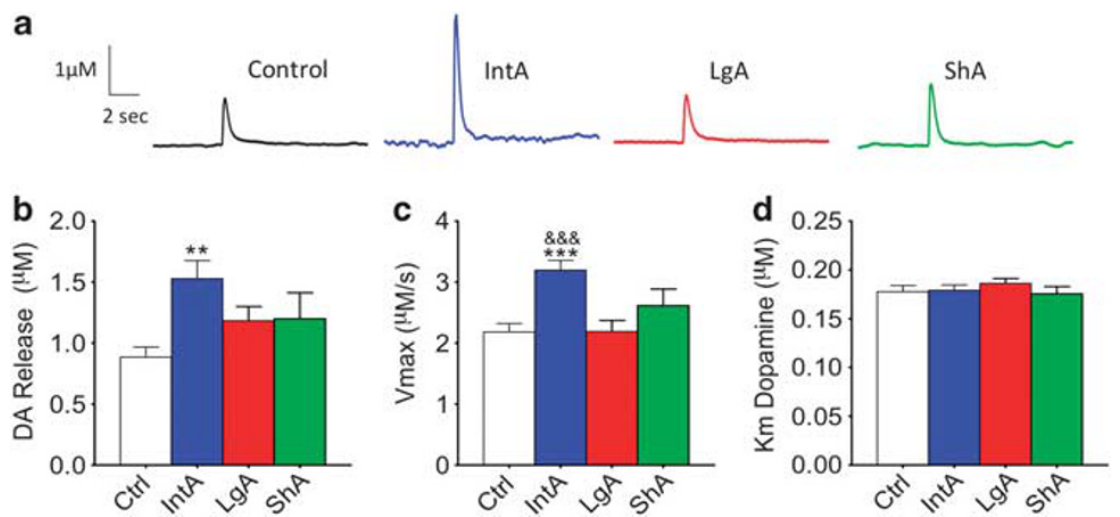

Figure 2 Differential effects of intermittent-access (IntA), short-access (ShA), and long-access (LgA) self-administration on presynaptic dopamine system kinetics. (a) Representative traces from control (left), IntA (center, left), LgA (center, right), and ShA (right) animals. Traces are represented as concentration $(\mu M)$ in dopamine (DA) over time. (b) Stimulated DA release in $\mu M$ across LgA, IntA, ShA, and control groups. (c) Maximal rate of DA uptake $\left(V_{\text {max }}\right)$ across groups. (d) Group data indicating that the $K_{m}$ of DA for the dopamine transporter is unchanged following all self-administration paradigms. $* * *<0.0$ I vs control; **** $p<0.00$ I vs control; ${ }^{\& \& \&} p<0.00$ I vs LgA.

and $\mathrm{b})$ and $V_{\max }\left(\mathrm{F}_{3,63}=8.397, p<0.0001\right.$; Figures $2 \mathrm{a}$ and c). Tukey's post hoc analysis revealed that stimulated release $(p<0.05$; Figure $2 \mathrm{~b})$ and $V_{\max }(p<0.05$; Figure $2 \mathrm{c})$ were both elevated in the IntA group as compared with controls. In addition, $V_{\max }$ was also elevated as compared with $\operatorname{LgA}$ $(p<0.001$; Figure $2 \mathrm{~b})$. Both $\operatorname{LgA}$ and ShA resulted in no significant change in either DA release or $V_{\max }$ as compared with control animals. There were no changes in any groups with regard to the affinity of DA for the DAT $\left(K_{\mathrm{m}}\right.$; Figure $\left.2 \mathrm{~d}\right)$. This indicates that pattern of cocaine interaction with the DAT has differential effects on the compensatory mechanisms associated with baseline functioning.

Differences in Intake (LgA vs ShA) Results in Decreased Cocaine Potency, While Differences in Temporal Pattern of Cocaine Intake (IntA vs ShA) Results in Increased Cocaine Potency at the DAT

FSCV in brain slices was used to determine cocaine potency over a concentration-response curve. ANOVA revealed a main effect of paradigm on cocaine potency $\left(\mathrm{F}_{3,68}=3.21\right.$, $p<0.01$; Figure 3 ). ShA resulted in no changes in cocaine potency as compared with controls (Figures 3a and c, black $v s$ green lines). When temporal pattern of cocaine selfadministration was kept the same, but there were differences in intake, cocaine potency was reduced (ShA vs LgA). This was expressed as a significant reduction in cocaine potency as measured by apparent $K_{\mathrm{m}}$ at the $30 \mu \mathrm{M}$ concentration in LgA $(p<0.001)$ vs ShA (Figure $3 c$, green $v s$ red curve). Conversely, when intake was kept the same, but there were differences in temporal pattern of selfadministration, cocaine potency was increased (IntA vs ShA). This was expressed as an increase in cocaine's ability to inhibit the DAT in the IntA group $(p<0.0001)$ as compared with ShA (Figure 3c, green $v s$ blue curve). A similar trend was present when groups were compared with control animals, where LgA resulted in decreased cocaine potency at the $30 \mu \mathrm{M}$ concentration $(p<0.01)$ and IntA resulted in enhanced potency for cocaine at the same
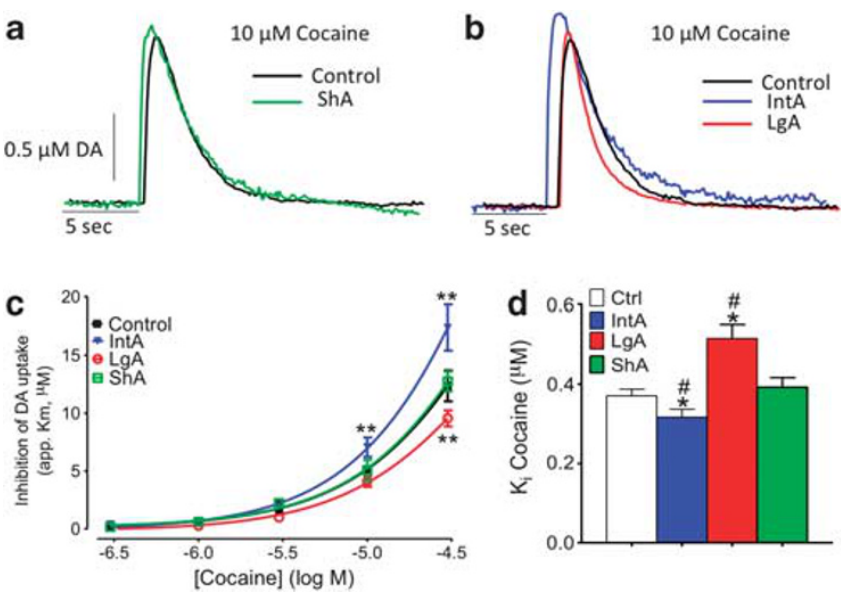

Figure 3 Intermittent-access (IntA) self-administration results in sensitization to the neurochemical effects of cocaine, while long-access ( LgA) results in tolerance. (a) Representative traces highlighting the uptake inhibition induced by $10 \mu \mathrm{M}$ cocaine in control (black) and short-access (ShA, green) animals. Traces are represented as concentration $(\mu M)$ in dopamine (DA) over time and smaller signals were shifted to the right in order to match the peak-height of the small signal to equivalent concentration of the larger signal. This allows for direct comparison of dopamine uptake in signals with different peak-heights. (b) Representative traces highlighting the uptake inhibition induced by $10 \mu \mathrm{M}$ cocaine in control (black), IntA (blue), and LgA (red) animals. (c) Cumulative cocaine $(0.3-30 \mu \mathrm{M})$ dose-response curves in slices containing the nucleus accumbens core. Cocaine potency is decreased following LgA, unchanged following ShA, and increased following IntA. (d) Group data of $K_{i}$ values for cocaine in control, LgA, ShA, and IntA groups. $K_{i}$ values are a measure of the concentration of drug at which $50 \%$ inhibition is achieved. $* P<0.5$ vs control; *** $p<0.0$ I vs control; and ${ }^{\#} p<0.0$ I vs ShA.

concentration $(p<0.01)$ (Figure $3 c$, red and blue curves $v s$ black curve).

$K_{\mathrm{i}}$ is a measure of the drug concentration that results in $50 \%$ uptake inhibition. This measurement can determine relative potencies between drugs at baseline as well as potency changes following drug self-administration. Kruskal-Wallis non-parametric analysis revealed a significant main effect of paradigm on $K_{\mathrm{i}} \quad\left(\chi_{4}^{2}=10.94\right.$, $p<0.05=10.53$; Figure $3 \mathrm{~d}$ and Supplementary Figure 1). 


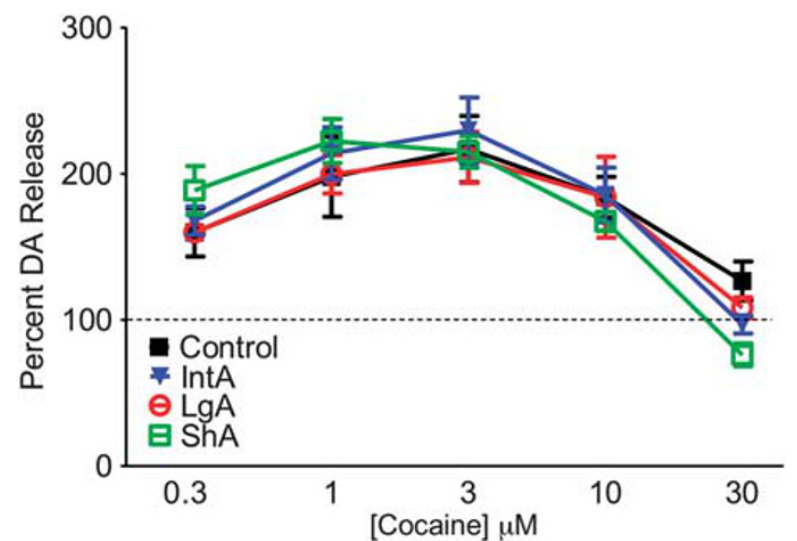

Figure 4 Cocaine-induced increases in dopamine (DA) did not differ across groups. Stimulated DA release was measured across a doseresponse curve for cocaine, and expressed as a percent pre-cocaine stimulated DA release. There were no differences in cocaine-induced DA elevations between control, intermittent-access (IntA), long-access (LgA), or short-access (ShA) cocaine self-administration groups.

Mann-Whitney analysis revealed that $K_{\mathrm{i}}$ was increased in LgA animals $(p<0.05)$ and reduced following IntA $(p<0.05)$ when compared with ShA. Further, $K_{\mathrm{i}}$ was increased in LgA animals $(p<0.05)$ and reduced following IntA $(p<0.05)$ when compared with control animals.

Cocaine-induced increases in peak height was assessed across all three groups to determine if this aspect of cocaine effects on DA was changed by self-administration. There were no significant differences between any of the groups tested (Figure 4).

\section{DISCUSSION}

These results demonstrate that the compensatory changes in DAT sensitivity to cocaine produced by self-administration are influenced by both the temporal pattern of cocaine consumption and the total amount of drug intake. When there was a sustained consumption pattern and intake was high $(\operatorname{LgA})$, tolerance to cocaine's effects at the DAT developed. Conversely, when intake was low and patterns of consumption were intermittent (IntA) sensitization of the DAT to cocaine occurred. Differences were also observed in baseline (pre-drug) DA measures, wherein IntA cocaine administration resulted in increased $V_{\max }$ and stimulated DA release, and LgA and ShA did not produce any changes. This is the first comparison of the effects ShA and LgA on cocaine potency directly at the DAT. Previous work showed increased measures of drug seeking, motivation to administer cocaine, and craving in LgA vs ShA animals (Koob, 1996; Koob and Le Moal, 1997; Orio et al, 2009; Patterson and Markou, 2003); however, these measures are probably not due to the pharmacological effects of cocaine at the DAT, which we demonstrate are reduced. Further, we found that IntA cocaine self-administration produced a sensitized cocaine response at the DAT, demonstrating that the pattern of self-administration has an integral role in the compensatory effects of the DAT to cocaine effects following extended use.

Here we show that $\operatorname{LgA}$ results in reduced cocaine potency, indicative of a neurochemical tolerance to cocaine at the DAT, in comparison to both ShA and naive control animals. The decreased cocaine potency following $\operatorname{LgA}$ is consistent with previous reports demonstrating similar changes following a number of different extended-access paradigms. In our hands and others, LgA results in neurobiological compensations characterized by decreased cocaine effects and blunted DA system function in the NAc (Hurd et al, 1989; Meil et al, 1995; Macey et al, 2004; Ferris et al, 2011, 2012; Calipari et al, 2012; but see Hooks et al, 1994; Zapata et al, 2003). Also, immediately following a 21day LgA cocaine self-administration while cocaine is still present, there are decreases in functional activity in the ventral and dorsal striatum, as well as a number of other brain regions, indicating that cocaine is less efficacious at activating these circuits (Macey et al, 2004). Previously, we examined a number of self-administration protocols, manipulating both frequency and duration of access, and regardless of time, animals given continuous cocaine access exhibited robust behavioral and neurochemical tolerance. Animals that administered cocaine for only 1 day of 40 injections and animals that underwent extended-access self-administration for 5 days (40 inj. per day) both exhibited the same effects as LgA self-administration (Ferris et al, 2011, 2012; Calipari et al, 2012). All of the previously tested procedures shared the property that animals could administer large amounts of cocaine for the duration of the session. Regardless of duration of testing, each of these groups that maintained high rates of responding over 5- to 6-h sessions showed neurochemical tolerance at the DAT when tested $24 \mathrm{~h}$ after the last self-administration session (Ferris et al, 2011, 2012; Calipari et al, 2012). Although ShA animals have a similar pattern of self-administration and can administer large amounts of drug, they can only do so for $2 \mathrm{~h}$. This results in significantly less drug consumption, leading to no change in cocaine potency. This suggests that total intake within sessions, and not pattern, is what is driving the tolerance to the pharmacological effects of cocaine at the DAT.

In contrast, here we show that the IntA cocaine self-administration procedure enhances cocaine potency, indicative of a sensitized neurochemical response to cocaine at the DAT relative to both ShA and naive controls. The IntA results are opposite to the well-documented decrease in cocaine potency after LgA shown here and in previous research (Hurd et al, 1989; Ferris et al, 2011, 2012; Calipari et al, 2012). Although behavioral sensitization to cocaine has been reported previously (Schmidt and Pierce, 2010), to our knowledge, this is the first demonstration of a sensitized cocaine response directly at the DAT following cocaine self-administration. It is possible that the increased potency may promote increased rewarding and reinforcing effects of cocaine as well as an increase in motivation to self-administer the drug, potentially leading to greater risk of compulsive or addictive-like cocaine intake. Indeed, Zimmer et al (2012) showed that motivation to selfadminister cocaine is significantly increased following IntA as compared with LgA and ShA cocaine self-administration.

The marked differences between ShA and IntA cannot be attributed to intake, as the animals administer similar amounts of drug over sessions. In addition, the session length was different between IntA (360 min) and ShA (120 min); however, we do not think that effects are due 
to session length differences, because $\operatorname{LgA}$ and IntA both had 360-min sessions. These groups exhibited opposite effects on DAT pharmacology; therefore, it seems that session time is not a contributing factor to the neurochemical effects. The increased effects of cocaine at the DAT seem to be due to the intermittent intake pattern of cocaine that likely leads to intermittent cocaine-DAT interactions. The $\operatorname{LgA}$ and IntA groups differ substantially in the total cocaine self-administered over the course of the current experiment; however, we would predict that LgA would still produce tolerance when total intake is limited to equal the IntA condition. In fact, we have shown that a single day of LgA (60 mg total), which approximates the IntA group's total intake of cocaine $(70 \mathrm{mg}$ total), produced robust tolerance (Ferris et al, 2011). Thus, the sensitized response was only seen in the group with intermittent cocaine access (IntA) and not in the matched intake groups that experienced sustained drug levels examined here (ie, ShA) and in previous work (ie LgA; Ferris et al, 2011).

Cocaine has been demonstrated to result in changes in DA release that are dependent on vesicular exocytosis (Venton et al, 2006); therefore, we wanted to confirm that changes in this process were not driving the observed changes in cocaine effects. DA release over the concentration-response curve for the compound was not changed in any of the groups, suggesting that it was cocaine-induced DAT inhibition, not increased/decreased DA release, causing the change in potency following IntA/ LgA. It is possible that allosteric modifications to the DAT protein itself are mediating the decreased potency following $\operatorname{LgA}$ and the increased potency following IntA. Indeed, the DAT has been shown to undergo glycosylation and phosphorylation (Foster et al, 2008; Chen et al, 2009). Therefore, it is possible that changes in the interaction of the DAT protein with downstream effectors responsible for these post-translational modifications are altered following self-administration in a way that changes the ability of ligands to bind to the DAT and inhibit the clearance of DA. In addition, the DAT has been shown to form oligomer complexes (Hastrup et al, 2003) and be modulated by receptors such as the D2 autoreceptor (Chen et al, 2013), which are two additional mechanisms that could have a role in the observed changes in cocaine potency.

There appears to be many neurochemical adaptations that occur following cocaine exposure, and how they interact to influence cocaine-induced DA overflow and behavior is as yet unclear. In addition to the sensitized effect at the DAT reported here, other systems appear to have a role in DA system sensitization, including glutamate (for a review, see Vanderschuren and Kalivas, 2000), GABA (for a review, see Steketee, 2005; Filip et al, 2006), and serotonin (Neumaier et al, 2002; Filip et al, 2010). Owing to the convergence of these systems and factors, which lead to the expression of behavioral sensitization, it remains to be determined whether increased DA overflow and behavioral effects would be seen after IntA self-administration. Further, demonstrations of sensitized DA overflow are more robust after 1-2 weeks of drug abstinence (Kalivas and Duffy, 1990, 1993); thus, it is possible that the changes observed here could be more robust following acute and prolonged withdrawal.
Here we demonstrate that whether tolerance or sensitization develops to specific effects of cocaine appears to depend on access conditions and the intake or pattern of administration, respectively. Pattern, total dose, and abstinence periods need to be taken into account when modeling the behavioral and neurochemical processes involved in addiction. Cocaine sensitization has been difficult to demonstrate in humans, suggesting that either sensitization does not occur in humans or that it occurs during a part of the addiction process that has not been studied extensively (Leyton and Vezina, 2013). It is possible that early in the drug abuse process administration of cocaine is intermittent, leading to a sensitized cocaine response that facilitates continued drug use, while after long-term use there is tolerance to acute drug effects, an effect that has been reported in human subjects (Mendelson et al, 1998; Reed et al, 2009). It is suggested that in humans, drug use may be based on limited availability, which may lead individuals to administer drug in an intermittent pattern (Ahmed et al, 2013). However, it has also been suggested that $\operatorname{LgA}$ self-administration is a model of the escalation that is reported with long-term abuse of the compound in humans (Dackis and O'Brien, 2001). Here we demonstrate tolerance to the neurochemical effects of cocaine following $\operatorname{LgA}$, suggesting that $\operatorname{LgA}$ may result in neurochemical changes consistent with human studies. In our previous work and currently accepted cocaine self-administration models, much emphasis has been placed on maximizing an animal's intake, with the thought that more intake results in greater neurochemical effects. Here we demonstrate that greater intake is not necessary to produce robust neurochemical effects and, in fact, continuous high intake early in the animal's self-administration history may produce marked tolerance to the drug. Thus, we highlight the importance of mimicking human patterns of administration in rodent models, as compensatory mechanisms associated with drug exposure are not only dependent on the drug and total intake but also on the pattern in which the drug is taken.

\section{FUNDING AND DISCLOSURE}

The authors declare no conflict of interest.

\section{ACKNOWLEDGEMENTS}

This work was funded by NIH Grants R01 DA021325, R01 DA030161 (SRJ), R01 DA014030 (DCSR), P50DA006634 (SRJ and DCSR), T32 DA007246 and F31 DA031533 (ESC), and K99 DA031791 (MJF).

\section{REFERENCES}

Addy NA, Daberkow DP, Ford JN, Garris PA, Wightman RM (2010). Sensitization of rapid dopamine signaling in the nucleus accumbens core and shell after repeated cocaine in rats. J Neurophysiol 104: 922-931.

Ahmed SH, Lenoir M, Guillem K (2013). Neurobiology of addiction vs drug use driven by lack of choice. Curr Opin Neurobiol 23: $1-7$.

Ahmed SH, Lin D, Koob GF, Parsons LH (2003). Escalation of cocaine self-administration does not depend on altered cocaine- 
induced nucleus accumbens dopamine levels. J Neurochem 86: 102-113.

Aragona BJ, Day JJ, Roitman MF, Cleaveland NA, Wightman RM, Carelli RM (2009). Regional specificity in the real-time development of phasic dopamine transmission patterns during acquisition of a cue-cocaine association in rats. Eur J Neurosci 30: $1889-1899$.

Blum K, Chen AL, Giordano J, Borsten J, Chen TJ, Hauser M et al (2012). The addictive brain: all roads lead to dopamine. J Psychoactive Drugs 44: 134-143.

Calipari ES, Ferris MJ, Melchior JR, Bermejo K, Salahpour A, Roberts DC et al (2012). Methylphenidate and cocaine selfadministration produce distinct dopamine terminal alterations. Addict Biol (in press).

Chen R, Daining CP, Sun H, Fraser R, Stokes SL, Leitges M et al (2013). Protein kinase $C \beta$ is a modulator of the dopamine D2 autoreceptor-activated trafficking of the dopamine transporter. I Neurochem 125: 663-672.

Chen R, Furman CA, Zhang MJ, Kim MN, Gereau RW, Leitges M et al (2009). Protein kinase C beta is a critical regulator of dopamine transporter trafficking and regulates the behavioral response to amphetamine in mice. J Pharmacol Exp Therap 328: 912-920.

Dackis CA, O'Brien CP (2001). Cocaine dependence: a disease of the brain's reward centers. J Subst Abuse Treat 21: 111-117.

Ferris MJ, Calipari ES, Mateo Y, Melchior JR, Roberts DC, Jones SR (2012). Cocaine self-administration produces pharmacodynamic tolerance: differential effects on the potency of dopamine transporter blockers, releasers, and methylphenidate. Neuropsychopharmacology 37: 1708-1716.

Ferris MJ, Mateo Y, Roberts DC, Jones SR (2011). Cocaine-insensitive dopamine transporters with intact substrate transport produced by self-administration. Biol Psychiatry 69: 201-207.

Filip M, Alenina N, Bader M, Przegaliński E (2010). Behavioral evidence for the significance of serotoninergic (5-HT) receptors in cocaine addiction. Addict Biol 15: 227-249.

Filip M, Frankowska M, Gołda A, Zaniewska M, Vetulani J, Przegaliński E (2006). Various GABA-mimetic drugs differently affect cocaine-evoked hyperlocomotion and sensitization. Eur J Pharmacol 541: 163-170.

Foster JD, Adkins SD, Lever JR, Vaughan RA (2008). Phorbol ester induced trafficking-independent regulation and enhanced phosphorylation of the dopamine transporter associated with membrane rafts and cholesterol. J Neurochem 105: 1683-1699.

Hastrup H, Sen N, Javitch JA (2003). The human dopamine transporter forms a tetramer in the plasma membrane: cross-linking of a cysteine in the fourth transmembrane segment is sensitive to cocaine analogs. J Biol Chem 278: 45045-45048.

Hooks MS, Duffy P, Striplin C, Kalivas PW (1994). Behavioral and neurochemical sensitization following cocaine self-administration. Psychopharmacology (Berl) 115: 265-272.

Hurd YL, Weiss F, Koob GF, And NE, Ungerstedt U (1989). Cocaine reinforcement and extracellular dopamine overflow in rat nucleus accumbens-an in vivo Microdialysis Study. Brain Res 498: 199-203.

Izenwasser S (2004). The role of the dopamine transporter in cocaine abuse. Neurotox Res 6: 379-383 (review).

Jones SR, Lee TH, Wightman RM, Ellinwood EH (1996). Effects of intermittent and continuous cocaine administration on dopamine release and uptake regulation in the striatum: in vitro voltammetric assessment. Psychopharmacology (Berl) 126: $331-338$.

Kalivas PW, Duffy P (1990). Effect of acute and daily cocaine treatment on extracellular dopamine in the nucleus accumbens. Synapse 5: 48-58.
Kalivas PW, Duffy P (1993). Time course of extracellular dopamine and behavioral sensitization to cocaine. I. Dopamine axon terminals. J Neurosci 13: 266-275.

Kalivas PW, McFarland K (2003). Brain circuitry and the reinstatement of cocaine-seeking behavior. Psychopharmacology (Berl) 168: 44-56.

Koob GF (1996). Hedonic valence, dopamine and motivation. Mol Psychiatry 1: 186-189.

Koob GF, Le Moal M (1997). Drug abuse: hedonic homeostatic dysregulation. Science 278: 52-58.

Kristensen AS, Andersen J, Jørgensen TN, Sørensen L, Eriksen J, Loland CJ et al (2011). SLC6 neurotransmitter transporters: structure, function, and regulation. Pharmacol Rev 63: 585-640 (review).

Lack CM, Jones SR, Roberts DC (2008). Increased breakpoints on a progressive ratio schedule reinforced by IV cocaine are associated with reduced locomotor activation and reduced dopamine efflux in nucleus accumbens shell in rats. Psychopharmacology (Berl) 195: 517-525.

Leyton M, Vezina P (2013). Striatal ups and downs: their roles in vulnerability to addictions in humans. Neurosci Biobehav Rev S0149-7634: 00019-5.

Liu Y, Morgan D, Roberts DCS (2007). Cross-sensitization of the reinforcing effects of cocaine and amphetamine in rats. Psychopharmacology (Berl) 195: 369-375.

Macey DJ, Rice WN, Freedland CS, Whitlow CT, Porrino LJ (2004). Patterns of functional activity associated with cocaine selfadministration in the rat change over time. Psychopharmacology (Berl) 172: 384-392.

Martin BJ, Naughton BJ, Thirtamara-Rajamani K, Yoon DJ, Han DD, Devries AC et al (2011). Dopamine transporter inhibition is necessary for cocaine-induced increases in dendritic spine density in the nucleus accumbens. Synapse 65: 490-496.

Mateo Y, Lack CM, Morgan D, Roberts DC, Jones SR (2005). Reduced dopamine terminal function and insensitivity to cocaine following cocaine binge self-administration and deprivation. Neuropsychopharmacology 30: 1455-1463.

Meil WM, Roll JM, Grimm JW, Lynch AM, See RE (1995). Tolerance-like attenuation to contingent and noncontingent cocaine-induced elevation of extracellular dopamine in the ventral striatum following 7 days of withdrawal from chronic treatment. Psychopharmacology (Berl) 118: 338-346.

Mendelson JH, Sholar M, Mello NK, Teoh SK, Sholar JW (1998). Cocaine tolerance: behavioral, cardiovascular, and neuroendocrine function in men. Neuropsychopharmacology 18: 263-271.

Neumaier JF, Vincow ES, Arvanitogiannis A, Wise RA, Carlezon WA Jr (2002). Elevated expression of 5-HT1B receptors in nucleus accumbens efferents sensitizes animals to cocaine. J Neurosci 22: 10856-10863.

Orio L, Edwards S, George O, Parsons LH, Koob GF (2009). A role for the endocannabinoid system in the increased motivation for cocaine in extended-access conditions. J Neurosci 29: 4846-4857.

Parsons LH, Justice JB Jr (1993). Serotonin and dopamine sensitization in the nucleus accumbens, ventral tegmental area, and dorsal raphe nucleus following repeated cocaine administration. J Neurochem 61: 1611-1619.

Paterson NE, Markou A (2003). Increased motivation for self-administered cocaine after escalated cocaine intake. NeuroReport 14: 2229-2232.

Peraile I, Torres E, Mayado A, Izco M, Lopez-Jimenez A, Lopez-Moreno JA et al (2010). Dopamine transporter down-regulation following repeated cocaine: implications for 3,4-methylenedioxymethamphetamine-induced acute effects and long-term neurotoxicity in mice. Br J Pharmacol 159: 201-211.

Reed SC, Haney M, Evans SM, Vadhan NP, Rubin E, Foltin RW (2009). Cardiovascular and subjective effects of repeated smoked 
cocaine administration in experienced cocaine users. Drug Alcohol Depend 102: 102-107.

Ritz MC, Lamb RJ, Goldberg SR, Kuhar MJ (1987). Cocaine receptors on dopamine transporters are related to self-administration of cocaine. Science 237: 1219-1223.

Roberts DC, Corcoran ME, Fibiger HC (1977). On the role of ascending catecholaminergic systems in intravenous self-administration of cocaine. Pharmacol Biochem Behav 6: 615-620.

Schmidt HD, Pierce RC (2010). Cocaine-induced neuroadaptations in glutamate transmission: potential therapeutic targets for craving and addiction. Ann N Y Acad Sci 1187: 35-75.

Steketee JD (2005). Cortical mechanisms of cocaine sensitization. Crit Rev Neurobiol 17: 69-86.

Stuber GD, Britt JP, Bonci A (2011). Optogenetic modulation of neural circuits that underlie reward seeking. Biol Psychiatry 71: 1061-1067.

Vanderschuren LJ, Kalivas PW (2000). Alterations in dopaminergic and glutamatergic transmission in the induction and expression of behavioral sensitization: a critical review of preclinical studies. Psychopharmacology (Berl) 151: 99-120.
Venton BJ, Seipel AT, Phillips PE, Wetsel WC, Gitler D, Greengard $P$ et al (2006). Cocaine increases dopamine release by mobilization of a synapsin-dependent reserve pool. J Neurosci 26: 3206-3209.

Wheeler RA, Carelli RM (2009). Dissecting motivational circuitry to understand substance abuse. Neuropharmacology 56(Suppl 1): 149-159.

Willuhn I, Wanat MJ, Clark JJ, Phillips PE (2010). Dopamine signaling in the nucleus accumbens of animals self-administering drugs of abuse. Curr Top Behav Neurosci 3: 29-71.

Yorgason JT, España RA, Jones SR (2011). Demon voltammetry and analysis software: analysis of cocaine-induced alterations in dopamine signaling using multiple kinetic measures. J Neurosci Methods 202: 158-164.

Zapata A, Chefer VI, Ator R, Shippenberg TS, Rocha BA (2003). Behavioural sensitization and enhanced dopamine response in the nucleus accumbens after intravenous cocaine self-administration in mice. Eur J Neurosci 17: 590-596.

Zimmer BA, Oleson EB, Roberts DCS (2012). The motivation to self-administer is increased after a history of spiking brain levels of cocaine. Neuropsychopharmacology 37: 1901-1910.

Supplementary Information accompanies the paper on the Neuropsychopharmacology website (http://www.nature.com/npp) 